\title{
Photoaffinity labeling of the lysosomal neuraminidase from bovine testis
}

\author{
Gijsbertus T.J. van der Horst ${ }^{1}$, Ursula Rose ${ }^{2}$, Reinhard Brossmer ${ }^{2}$ and Frans W. Verheijen ${ }^{1}$ \\ ${ }^{1}$ Department of Cell Biology and Genetics, Erasmus University, Rotterdam, The Netherlands and ${ }^{2}$ Department of Biochemistry II, \\ University of Heidelberg, Heidelberg, Germany
}

Received 19 September 1990; revised version received 19 October 1990

\begin{abstract}
ASA-NeuAc2en, a photoreactive arylazide derivative of sialic acid, is shown to be a powerful competitive inhibitor of lysosomal neuraminidase from bovine testis $\left(K_{\mathrm{i}} \approx 21 \mu \mathrm{M}\right)$. Photoaffinity labeling and partial purification of preparations containing this lysosomal neuraminidase activity result in specifically and non-specifically labeled polypeptides. Only labeling in a $55 \mathrm{kDa}$ polypeptide is found to be specific, since it could be prevented by the competitive neuraminidase inhibitor NeuAc2en. We conclude that the $55 \mathrm{kDa}$ polypeptide in the bovine testis $\beta$-galactosidase/neuraminidase/protective protein complex contains the catalytic site of neuraminidase.
\end{abstract}

Neuraminidase; Sialidase; Lysosomal; Photoaffinity labeling

\section{INTRODUCTION}

In the inherited metabolic disorder sialidosis the intralysosomal degradation of sialoglycoconjugates is impaired due to a deficiency of the acid hydrolase neuraminidase (sialidase EC 3.2.1.18) [1-3]. In galactosialidosis a combined deficiency of neuraminidase and $\beta$-galactosidase exists $[4,5]$. The latter disease is caused by a mutation in the 'protective protein', which protects $\beta$-galactosidase from intralysosomal degradation and is essential for neuraminidase activity [6]. No information is available about the molecular defect in sialidosis.

Active neuraminidase has been isolated from bovine testis and human placenta as a high molecular mass complex with $\beta$-galactosidase and the protective protein $[7,8]$. Partial disruption of the complex with KSCN results in the dissociation of the neuraminidase polypeptide from the complex. The unassociated neuraminidase polypeptide has no enzymatic activity [9]. Reconstitution experiments have shown that, in vitro, association of the neuraminidase polypeptide with the protective protein generates a labile form of neuraminidase activity. This activity is stabilized by $\beta$-galactosidase [10]. Most likely the neuraminidase polypeptide contains the catalytic site.

Correspondence address: G.T.J. van der Horst, Dept. of Cell Biology and Genetics, Erasmus University, PO Box 1738, 3000 DR Rotterdam, The Netherlands

Abbreviations: ASA-NeuAc2en, 5- $N$-acetyl-9-(4-azidosalicoylamido)-2-deoxy-2,3-didehydroneuraminic acid; IASA-NeuAc2en, 5- $\mathrm{N}$ acetyl-9-(4-iodoazidosalicoylamido)-2-deoxy-2,3-didehydroneuraminic acid; NeuAc2en, 2-deoxy-2,3-didehydro-5- $\mathrm{N}$-acetylneuraminic acid; MU-NeuAc, 2- $\alpha$-(4-methylumbelliferyl)-5- $N$-acetylneuraminic acid; SDS, sodium dodecyl sulfate
To investigate the catalytic site of neuraminidases, we have recently synthesized the radioiodinatable, photoreactive affinity probe ASA-NeuAc2en. This probe was successfully used to label the active site of a bacterial neuraminidase [11]. In the present study we have used ASA-NeuAc2en to label the neuraminidase polypeptide in the lysosomal $\beta$-galactosidase/neuraminidase/protective protein complex from bovine testis.

\section{MATERIALS AND METHODS}

\subsection{Materials}

ASA-NeuAc2en and $\left[{ }^{125}\right.$ I]IASA-NeuAc2en were prepared as described previously [11]. The $\beta$-galactosidase/neuraminidase/protective protein complex was purified from bovine testis as reported by Verheijen et al. [7].

\subsection{Photoaffinity labeling and further purification of bovine testis neuraminidase

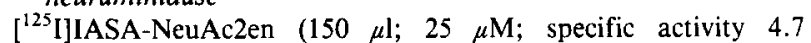 $\mathrm{Ci} / \mathrm{mmol}$ ) was dried under $\mathrm{N}_{2}$ in absence or presence of $20 \mu 1100 \mathrm{mM}$ NeuAc2en. The dried material was dissolved in $200 \mu 1$ bovine testis complex $(200 \mu \mathrm{g}$; specific activity $113 \mathrm{mU} / \mathrm{mg})$ in $20 \mathrm{mM}$ sodium acetate, $100 \mathrm{mM} \mathrm{NaCl}$, pH 5.2 (Buffer A), containing $1 \%$ Triton $\mathrm{X}-100$ and incubated for $5 \mathrm{~min}$ at room temperature (final concentra- tion of [ ${ }^{125} \mathrm{I}$ ]IASA-NeuAc2en $18.75 \mu \mathrm{M}$ ). After cooling on ice for 30 $s$ and addition of $\beta$-mercaptoethanol (final concentration $100 \mathrm{mM}$ ), samples were exposed to $254 \mathrm{~nm}$ UV-light for 1 min at a distance of $1 \mathrm{~cm}$. Unbound label was removed by rapid gel filtration through a Sephadex G-50 medium spin column [12], equilibrated with buffer A containing $1 \%$ Triton $\mathrm{X}-100$. Under these conditions, no detectable UV-induced protein-protein crosslinking or ASA-NeuAc2en-specific inactivation of neuraminidase was observed. \\ After photoaffinity labeling, neuraminidase was further purified by sucrose density gradient centrifugation on a linear $20-40 \%$ sucrose gradient in buffer $\mathrm{A}\left(15 \mathrm{~h} ; 150000 \times g ; 4^{\circ} \mathrm{C}\right)$. Fractions containing neuraminidase activity were pooled. Protein was precipitated with $10 \%$ TCA and analyzed on a $11 \%$ SDS-polyacrylamide gel as describ- ed by Laemmli [13]. Photoaffinity-labeled proteins were visualized by autoradiography of the dried gel.}




\subsection{Enzymatic assays}

Neuraminidase and $\beta$-galactosidase activities were measured with the corresponding 4-methylumbelliferyl substrates as described [14]. Kinetic experiments were performed as previously reported [11] in either neuraminidase assay buffer (125 mM sodium acetate, $\mathrm{pH} 4.3$ ) or in buffer A. Protein concentrations were determined according to Lowry et al. [15].

\section{RESULTS AND DISCUSSION}

To generate optimal conditions for successful photoaffinity labeling of a mammalian lysosomal neuraminidase two considerations were made. First, mammalian neuraminidases are known to be very labile [16]. We have performed our experiments with the $\beta$-galactosidase/neuraminidase/protective protein complex isolated from bovine testis [7], since among the purified lysosomal neuraminidases it is the most stable form available. Secondly, photoaffinity labeling experiments with complex protein mixtures are often hindered by a large extent of non-specific labeling [17]. To reduce non-specific labeling we have used the scavenger $\beta$ mercaptoethanol [17]. To increase the ratio between specifically and non-specifically labeled proteins we have partially purified the neuraminidase after the labeling reaction.

ASA-NeuAc2en is a derivative of NeuAc2en, a potent competitive inhibitor of mammalian neuraminidases [18]. To investigate whether introduction of the photoreactive group in NeuAc2en has reduced its affinity for the bovine testis neuraminidase, $K_{\mathrm{i}}$ values for ASA-NeuAc2en and NeuAc2en were compared in kinetic experiments with the purified complex. Under standard assay conditions the $K_{\mathrm{m}}$ for 4-MU-NeuAc is $115 \mu \mathrm{M}$. The $K_{\mathrm{i}}$ values for ASA-NeuAc2en and NeuAc2en are 21 and $16 \mu \mathrm{M}$, respectively (Fig. 1A). This demonstrates that despite the presence of the arylazide group ASA-NeuAc2en is efficiently recognized by the bovine testis neuraminidase.

To avoid the risk of protein loss due to buffer changes and $\mathrm{pH}$ shifts, we have tested whether the label-

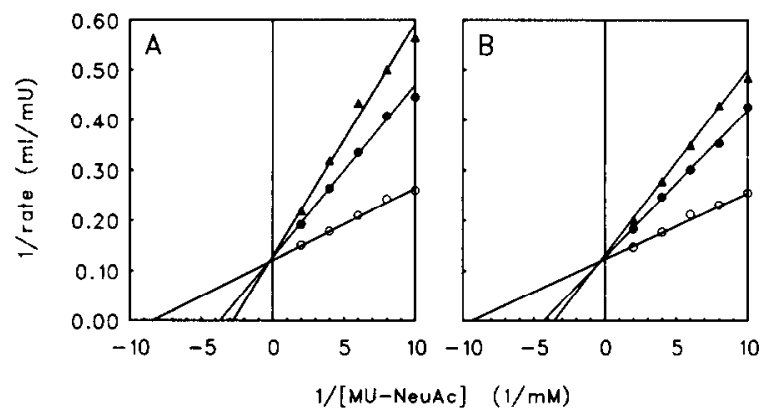

Fig. 1 Inhibition kinetics of lysosomal bovine testis neuraminidase with NeuAc2en and ASA-NeuAc2en. Purified bovine testis complex $(8 \mathrm{mU} / \mathrm{ml})$ was incubated with varying concentrations of 4-MUNeuAc at pH 4.3 (panel A) or 5.2 (panel B) in absence of inhibitor

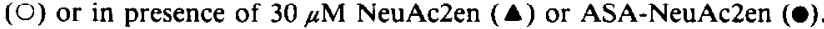
The enzyme activity is plotted as double reciprocal against the substrate concentration.

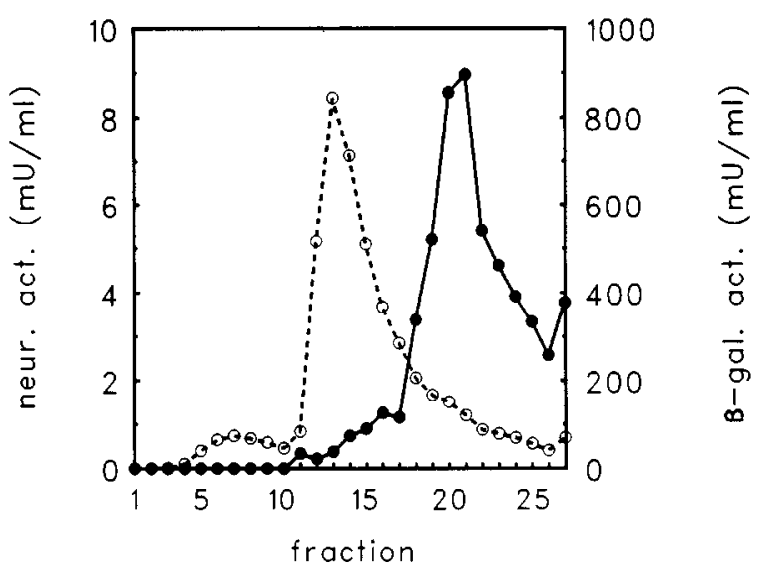

Fig. 2 Sucrose density centrifugation of $200 \mu \mathrm{g}$ purified bovine testis $\beta$-galactosidase/neuraminidase/protective protein complex on a $20-40 \%$ linear sucrose gradient. (๑) Neuraminidase activity, (O--O) $\beta$-galactosidase activity.

ing reaction can be performed in the buffer used for the purification of the complex. When kinetic experiments were performed in buffer A (Fig. 1B), the affinity of bovine testis neuraminidase for 4-MU-NeuAc $\left(K_{\mathrm{m}} 104\right.$ $\mu \mathrm{M})$, ASA-NeuAc2en $\left(K_{\mathrm{i}} 25 \mu \mathrm{M}\right)$, and NeuAc2en $\left(K_{\mathrm{i}} 16\right.$ $\mu \mathrm{M})$ is not altered. Also, addition of the scavenger $\beta$ mercaptoethanol had no effect on the affinity (data not shown). The ability to perform the photoaffinity labeling reaction in buffer A greatly facilitates the further purification of neuraminidase after labeling.

For glycoprotein preparations from human placenta sucrose density gradient centrifugation has been used to

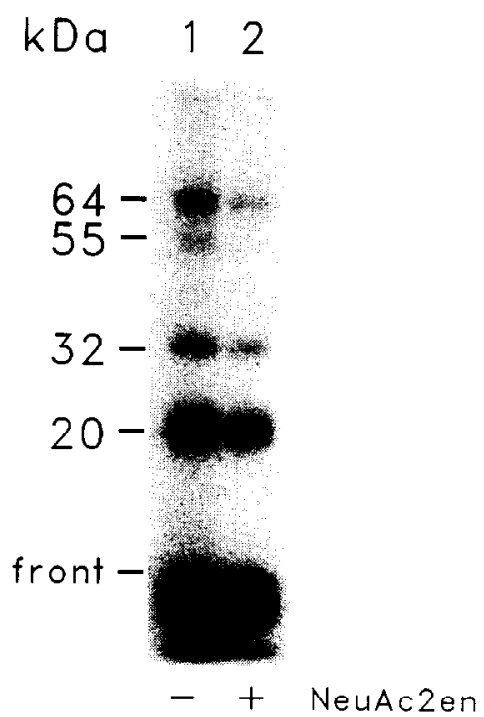

Fig. 3 Photoaffinity labeling of bovine testis neuraminidase. Bovine testis $\beta$-galactosidase/neuraminidase/protective protein complex (200 $\mu \mathrm{g})$ was photoaffinity-labeled with [ ${ }^{125}$ I]IASA-NeuAc2en. Neuraminidase was partially purified by sucrose density centrifugation and analyzed on a $11 \%$ SDS-polyacrylamide gel. Lane 1: labeling performed in absence of inhibitor; lane 2: labeling performed in presence of $10 \mathrm{mM}$ NeuAc2en 
separate complexes with neuraminidase and $\beta$-galactosidase activity from complexes only containing $\beta$ galactosidase activity [8]. Fig. 2 shows the distribution of neuraminidase and $\beta$-galactosidase activity after centrifugation of $200 \mu \mathrm{g}$ bovine testis complex on a 20 $40 \%$ linear sucrose gradient. With this method the neuraminidase activity can be separated from the majority of $\beta$-galactosidase activity.

Purified bovine testis complex was photoaffinitylabeled with $\left[{ }^{125}\right.$ I]IASA-NeuAc2en and neuraminidase was further purified by sucrose density centrifugation. After SDS-polyacrylamide gel electrophoresis, radiolabeled proteins with molecular masses of $64,55,32$ and $20 \mathrm{kDa}$ were observed (Fig. 3, lane 1). The pattern corresponds with that of the purified complex after Coomassie staining [7]. The $64 \mathrm{kDa}$ protein is $\beta$-galactosidase, whereas the 32 and $20 \mathrm{kDa}$ proteins are the two polypeptides of the heterodimeric protective protein $[7,19]$. When labeling is performed in the presence of the competitive inhibitor NeuAc2en at a concentration that prevents specific labeling (Fig. 3, lane 2), only labeling of the $55 \mathrm{kDa}$ protein is prevented. Identical results were obtained when total glycoprotein preparations from bovine testis were used, providing that after labeling neuraminidase was further purified by isolation of the complex and sucrose density gradient centrifugation (data not shown). This demonstrates that ASA-NeuAc2en can also be used with less pure neuraminidase preparations. We conclude that the $55 \mathrm{kDa}$ protein is the neuraminidase polypeptide, specifically labeled at the active site. This protein is smaller than the $66 \mathrm{kDa}$ neuraminidase polypeptide observed in human placenta [10]. A comparative biochemical study of both polypeptides is in progress.

After the successful application of ASA-NeuAc2en for the labeling of a bacterial neuraminidase [11], we have now extended its use for the labeling of a mammalian lysosomal neuraminidase. ASA-NeuAc2en enables further localization of catalytic site sequences at the peptide level [11]. This opens new possibilities for obtaining detailed information about the active site of the lysosomal neuraminidase and the molecular defect in sialidosis.

\section{REFERENCES}

[1] Cantz, M., Gehler, J. and Spranger, J. (1977) Biochem. Biophys. Res. Commun. 74, 732-738.

[2] Lowden, J.A. and O'Brien, J.S. (1979) Am. J. Hum. Genet. 31, $1-18$.

[3] O'Brien, J.S. (1982) in: Genetic Errors of Glycoprotein Metabolism (Durand, P. and OBrien, J.S. eds) pp. 33-48, Springer.

[4] Wenger, D.A., Tarby, T.J. and Wharton, C. (1978) Biochem. Biophys. Res. Commun. 82, 589-595

[5] Andria, G., Strisciuglio, P., Pontarelli, G., Sly, W.S. and Dodson, W.E. (1981) in: Sialidases and Sialidoses: Perspectives in Inherited Mctabolic Discascs (Tettamanti, G., Durand, P. and Di Donato, S. eds) vol. 4, pp. 379-395, Ermes, Milan, Italy.

[6] d'Azzo, A., Hoogeveen, A.T., Reuser, A.J.J., Robinson, D. and Galjaard, H. (1982) Proc. Natl. Acad. Sci. USA 79, 4535-4539.

[7] Verheijen, F.W., Brossmer, R. and Galjaard, H. (1982) Biochem. Biophys. Res. Commun. 108, 868-875.

[8] Verheijen, F.W., Palmeri, S., Hoogeveen, A.T. and Galjaard, H. (1985) Eur. J. Biochem. 149, 315-321.

[9] Verheijen, F.W., Palmeri, S. and Galjaard, H. (1987) Eur. J. Biochem. 162, 63-67.

[10] van der Horst, G.T.J., Galjart, N.J., d'Azzo, A., Galjaard, H. and Verheijen, F.W. (1989) J. Biol. Chem. 264, 1317-1322.

[11] van der Horst, G.T.J., Mancini, G.M.S., Brossmer, R., Rose, U and Verheijen, F.W. (1990) J. Biol. Chem. 265, 10801-10804.

[12] Penefsky, H.S. (1977) J. Biol. Chem. 252, 2891-2899.

[13] Laemmli, U.K. (1970) Nature 227, 680-685.

[14] Galjaard, H. (1980) Genetic Metabolic Diseases: Early Diagnosis and Prenatal Analysis, Elsevier, Amsterdam.

[15] Lowry, O.H., Rosebrough, N.J., Farr, A.L. and Randall, R.J. (1951) J. Biol. Chem. 193, 265-275.

[16] Conzclmann, E. and Sandhoff, K. (1987) Adv. Enzymol. Relat. Areas Mol. Biol. 60, 89-217.

[17] Bayley, H. (1983) in: Photogenerated Reagents in Biochemistry and Molecular Biology (Work, T.S. and Burdon, R.H. eds) Lab. Techn. in Biochem. and Mol. Biol., vol. 12, pp. 66-111, Elsevier, Amsterdam.

[18] Corfield, A.P., Michalski, J.C. and Schauer, R. (1981) in: Sialidases and Sialidoses: Perspectives in Inherited Metabolic Diseases (Tettamanti, G., Durand, P. and Di Donato, S. eds) vol. 4, pp. 1-70, Ermes, Milan, Italy.

[19] Galjart, N.J., Gillemans, N., Harris, A., van der Horst, G.T.J., Verheijen, F.W., Galjaard, H. and d'Azzo, A. (1988) Cell 54, 755-764. 